\title{
Neonatal Developmental Venous Anomalies: Clinicoradiologic Characterization and Follow-Up
}

\author{
(D) A.F. Geraldo, (D) S.S. Messina, (DD. Tortora, (D)A. Parodi, (D) M. Malova, (D) G. Morana, (D). Gandolfo, (D)A. D’Amico,
}

(D)E. Herkert, (DP. Govaert, (D) L.A. Ramenghi, (D)A. Rossi, and (D). Severino

\begin{abstract}
BACKGROUND AND PURPOSE: Although developmental venous anomalies have been frequently studied in adults and occasionally in children, data regarding these entities are scarce in neonates. We aimed to characterize clinical and neuroimaging features of neonatal developmental venous anomalies and to evaluate any association between MR imaging abnormalities in their drainage territory and corresponding angioarchitectural features.
\end{abstract}

MATERIALS AND METHODS: We reviewed parenchymal abnormalities and angioarchitectural features of 41 neonates with developmental venous anomalies (20 males; mean corrected age, 39.9 weeks) selected through a radiology report text search from 2135 neonates who underwent brain MR imaging between 2008 and 2019. Fetal and longitudinal MR images were also reviewed. Neurologic outcomes were collected. Statistics were performed using $\chi^{2}$, Fisher exact, Mann-Whitney $U$, or $t$ tests corrected for multiple comparisons.

RESULTS: Developmental venous anomalies were detected in $1.9 \%$ of neonatal scans. These were complicated by parenchymal/ventricular abnormalities in $15 / 41$ cases (36.6\%), improving at last follow-up in 8/10 (80\%), with normal neurologic outcome in $9 / 14$ $(64.2 \%)$. Multiple collectors $(P=.008)$ and larger collector caliber $(P<.001)$ were significantly more frequent in complicated developmental venous anomalies. At a patient level, multiplicity $(P=.002)$ was significantly associated with the presence of $\geq 1$ complicated developmental venous anomaly. Retrospective fetal detection was possible in $3 / 11$ subjects (27.2\%).

CONCLUSIONS: One-third of neonatal developmental venous anomalies may be complicated by parenchymal abnormalities, especially with multiple and larger collectors. Neuroimaging and neurologic outcomes were favorable in most cases, suggesting a benign, self-limited nature of these vascular anomalies. A congenital origin could be confirmed in one-quarter of cases with available fetal MR imaging.

ABBREVIATIONS: $\mathrm{CCM}=$ cerebral cavernous malformation; c-DVA = complicated developmental venous anomaly; $c$ US = cerebral ultrasound; CVMS = cerebrofacial venous metameric syndrome; DVA = developmental venous anomaly; $\mathrm{u}$-DVA = uncomplicated developmental venous anomaly

D evelopmental venous anomalies (DVAs) are the most frequently diagnosed intracranial vascular malformations, often encountered as incidental neuroimaging findings. ${ }^{1,2}$ On MR imaging, DVAs are recognized on postcontrast T1WI as radially oriented veins with a "caput medusae" pattern converging into 1 (or rarely more) dilated venous collector. ${ }^{3,4}$ These features may be also detected on precontrast MR images, ${ }^{3-5}$ especially if $\mathrm{T}^{*}{ }^{*}$ weighted sequences such as high-resolution SWI are included in

Received April 15, 2020; accepted after revision August 6.

From the Neuroradiology Unit (A.F.G.), Centro Hospitalar de Vila Nova de Gaia/ Espinho, Vila Nova de Gaia, Portugal; Neuroradiology Unit (A.F.G., D.T., G.M., A.R., M.S.), Neonatal Intensive Care Unit (A.P., M.M., L.A.R.), and Interventional Unit (C.G.), IRCCS Istituto Giannina Gaslini, Genova, Italy; Radiology Unit (S.S.M.), Casa di Cura Regina Pacis, Palermo, Italy; Dipartimento di Scienze Biomediche Avanzate (A.D.), Universita' Federico II, Napoli, Italy; and Division of Neonatology (E.H., P.G.), Department of Paediatrics, Erasmus University Medical Centre, Rotterdam, the Netherlands.; and

This work was supported by funds from "Ricerca Corrente Disordini Neurologici e Muscolari (Linea 5)" of the Italian Ministry of Health and the Compagnia di San Paolo (ROL 20573). the protocol. ${ }^{5}$ In addition, DVAs may be occasionally recognized in utero using fetal MR imaging. ${ }^{6}$

DVAs are usually considered benign anatomic variants. ${ }^{7}$ However, they represent areas of venous fragility that can become symptomatic through diverse pathomechanisms. ${ }^{8,9}$ Indeed, DVAassociated brain abnormalities are frequently depicted, including-

Paper previously presented, in part, as an oral communication at: Italian Congress of Pediatric Neuroradiology, October 11-13, 2018; Brescia, Italy.

All procedures performed in the studies involving human participants were in accordance with the ethical standards of 1964 Helsinki Declaration and its later amendments or comparable ethical standards. Informed consent was waived by the institutional research committee.

Please address correspondence to Andrea Rossi, MD, Neuroradiology Unit, IRCCS Istituto Giannina Gaslini, Via Gaslini 5, Genova 16148 Italia; e-mail: andrearossi@gaslini.org; @AndreaRossi_NRX; @MSavinaSeverino

\footnotetext{
- Indicates open access to non-subscribers at www.ajnr.org

$\equiv$ Indicates article with supplemental on-line appendix and table.

Indicates article with supplemental on-line photos.

http://dx.doi.org/10.3174/ajnr.A6829
} 
but-not limited-to sporadic cerebral cavernous malformations (CCMs). ${ }^{8-16}$ Moreover, a higher prevalence of DVAs has been described in patients with different pathologies and/or genetic conditions. $^{17-21}$

Although DVAs are widely described and characterized in adults, they remain under-reported in the pediatric population. Indeed, there are noticeably fewer studies focusing exclusively on DVAs in this age group, especially in the neonatal period. ${ }^{17,18,21-24}$ In particular, the largest case series of neonatal DVAs described so far included 14 neonates, mostly detected using ultrasound during routine scanning for other reasons, ${ }^{22}$ with limited information on the prevalence and perinatal characteristics of these vascular abnormalities, including complications and longitudinal evolution. Moreover, additional data on neonatal and fetal DVAs would be of great interest because there is an ongoing debate regarding their congenital or postnatal etiology. ${ }^{25}$

In this study, we aimed to describe the pre- and postnatal appearance of DVAs and associated brain anomalies in a relatively large single-center group of neonates, providing information on their imaging and clinical follow-up. In addition, we tested a possible association between parenchymal and ventricular abnormalities in the drainage territory of neonatal DVAs and their angioarchitectural features.

\section{MATERIALS AND METHODS Population}

After institutional review board approval, 1 pediatric neuroradiologist (M.S.) searched in the radiology information system of a tertiary pediatric institution (IRCCS Istituto Giannina Gaslini, Genoa, Italy) for reports of brain MR imaging studies performed in subjects up to 28 days of corrected age containing the term "developmental venous anomaly," during a 12-year period (January 2008 to December 2019). During this period, 2135 neonates underwent brain MR imaging. All procedures performed in the studies involving human participants were in accordance with the ethical standards of 1964 Helsinki Declaration and its later amendments or comparable ethical standards. Informed consent was waived by the institutional research committee.

\section{MR Imaging Technique and Image Analysis}

Neonates were scanned on 1.5T or 3T MR imaging units with different imaging protocols, all including at least T1WI, T2WI, DWI, and $\mathrm{T} 2{ }^{\star} \mathrm{WI}$ (either gradient recalled-echo or SWI) sequences. Gadolinium-based contrast agents were injected only if clinically indicated. Neonates were fed before the MR imaging examination to achieve spontaneous sleep, with mild oral midazolam sedation $(0.1 \mathrm{mg} / \mathrm{kg})$ in case of head movements, and were breathing spontaneously during the examination.

Brain MR imaging studies were reviewed by 2 pediatric neuroradiologists (M.S. and A.F.G. with 10 and 5 years of experience, respectively), who confirmed the diagnosis and evaluated the presence of DVA-related mechanical compression of adjacent structures, draining vein thrombosis, and/or parenchymal abnormalities within the drainage territory. The latter included any of the following: increased T2 signal of surrounding WM, foci of restricted diffusion, hemorrhage, $\mathrm{CCM},{ }^{26}$ malformations of cortical development, or calcifications (defined as focal areas of hyperintensity on SWI phase images in right-handed MR imaging systems or hyperdensity on head CT scans). Microhemorrhages were distinguished from type IV CCMs on the basis of their evolution on imaging. Indeed, vessels of CCMs have a tendency to leak and bleed, thus frequently increasing or stabilizing in size with time, while microhemorrhages typically present a regular evolution of hemoglobin degradation with a faster reduction in size and/or complete regression.

Subjects with $\geq 1$ associated abnormality were considered to have complicated DVAs (c-DVA group), and the remainder, uncomplicated DVAs (u-DVA group).

Additionally, we registered the number of DVAs per patient as well as the corresponding angioarchitecture features: ${ }^{3,11}$ direction of drainage, number of collector veins, and mean collector caliber (defined as the caliber of the collector vein in case of a single collector or the mean of all collector calibers in case of multiple collectors, measured on axial $\mathrm{T} 2^{\star} \mathrm{WI}$ ). Multiple collectors were defined as $\geq 2$ draining veins. Fetal MR imaging, neonatal cerebral ultrasound (cUS), DSA, and follow-up MR imaging were reviewed when available.

Imaging findings at last MR imaging follow-up were classified as interval improvement, progression, stability, or mixed evolution.

Discrepancies were resolved by a third pediatric neuroradiologist (A.R. with 25 years of experience).

\section{Clinical Data}

Data on sex, pregnancy history, gestational age at birth, cause of prematurity, type of delivery, Apgar scores, corrected age at first MR imaging, and imaging indications were obtained from the electronic clinical records. For neonates with c-DVAs, additional data including treatment, age at last clinical assessment, and neurologic outcome (graded as normal, mild, moderate, or severe impairment) were also registered.

\section{Statistical Analysis}

Quantitative data were presented as median and interquartile range, and categoric data, as frequencies and percentages. Fisher exact, $\chi^{2}$, and independent samples Student $t$ tests were used to compare clinical characteristics between patient groups with c-DVAs and uDVAs. Fisher exact, $\chi^{2}$, and Mann-Whitney $U$ tests were used to compare angioarchitectural characteristics and associated parenchy$\mathrm{mal} /$ ventricular abnormalities between individual complicated and uncomplicated DVAs. All results were corrected for multiple-comparison testing using the Bonferroni correction method. Statistical significance was reached if the $P$ value was $<0.05 / \mathrm{k}$, where $\mathrm{k}$ indicates the number of tests, resulting in thresholds for statistical significance of $P<.0045$ and .0083 for patient and DVA levels of comparison, respectively. Statistical analyses were performed using SPSS Statistics software, Version 24.0 (IBM).

\section{RESULTS}

\section{Neonatal Imaging Features}

Forty-one neonates with DVAs were retrieved by a report search and confirmed by image review (20 males; mean corrected age at first MR imaging, 39.9 weeks; range, 33-44 weeks), corresponding to a real-world MR imaging DVA detection of 1.9\% (41/ $2135)$ in a tertiary pediatric center. Neonates were preterm in $46.3 \%$ of cases $(n=19)$. Brain MR imaging was obtained on a $3 \mathrm{~T}$ scanner in 22 cases $(53.7 \%)$. SWI and postcontrast T1WI were acquired in 38 (92.7\%) and 7 cases (17.1\%), respectively.

AJNR Am J Neuroradiol 41:2370-76 Dec 2020 www.ajnr.org 
Table 1: Location and angioarchitecture characteristics of developmental venous anomalies

\begin{tabular}{lcccc}
\hline & $\begin{array}{c}\text { Total } \\
(\boldsymbol{n}=\mathbf{5 8})\end{array}$ & $\begin{array}{c}\text { Complicated DVA } \\
(\boldsymbol{n}=\mathbf{2 1})(36.2 \%)\end{array}$ & $\begin{array}{c}\text { Uncomplicated DVA } \\
(\boldsymbol{n}=37)(63.2 \%)\end{array}$ & $\begin{array}{c}\boldsymbol{P} \\
\text { Value }^{\mathrm{a}}\end{array}$ \\
\hline Location (\%) & & & & .44 \\
Frontal & $24(41.4)$ & $9(42.9)$ & $15(40.5)$ & \\
Parieto-occipital & $16(27.7)$ & $6(28.6)$ & $10(27)$ & \\
Temporal & $8(13.8)$ & $3(14.3)$ & $5(13.5)$ & \\
Basal ganglia/thalami & $5(8.6)$ & $0(0)$ & $5(13.5)$ & \\
Brain stem & $2(3.4)$ & $1(4.8)$ & $1(2.7)$ & .34 \\
Cerebellum & $3(5.2)$ & $2(9.5)$ & $1(2.7)$ & .59 \\
Infratentorial (\%) & $5(8.6)$ & $3(14.3)$ & $2(5.4)$ & $.008^{\mathrm{b}}$ \\
Right side (\%) & $33(56.9)$ & $13(61.9)$ & $20(54.1)$ & \\
Multiple collectors (\%) & $9(15.5)$ & $7(33.3)$ & $2(5.4)$ & $.001^{\mathrm{b}}$ \\
Main collector caliber & $1.6(1.18-2.10)$ & $2.1(1.95-2.30)$ & $1.2(1-1.6)$ & \\
$\quad$ (median) (IQR) (mm) & & & & \\
Drainage (\%) & & $11(52.4)$ & $20(54.1)$ & \\
Deep & $31(53.4)$ & $6(28.6)$ & $13(35.1)$ & \\
Superficial & $19(32.8)$ & $4(19)$ & $4(10.8)$ & \\
Both & $8(13.8)$ & &
\end{tabular}

Note:-IQR indicates interquartile range.

${ }^{a} P$ values for group comparisons were determined by $\chi^{2}$ or Fisher exact tests for categoric variables or by MannWhitney $U$ tests for continuous variables, as appropriate.

${ }^{b}$ Value statistically significant (statistical significance was set at $P<.0083$ after Bonferroni correction for multiple comparisons). were affected by cerebrofacial venous metameric syndrome (CVMS). The On-line Table summarizes clinicoradiologic associations. In particular, at-term birth $(P=.02)$, higher gestational age $(P=.05)$, and imaging indications other than "preterm screening" $(P=$ $.005)$ were significantly more frequent in the c-DVA group but did not reach statistical significance after adjusting for multiple comparisons. Moreover, multiple DVAs as well as additional craniofacial vascular lesions were also more common in patients with c-DVAs $(P=$ .002 and $P=.02$, respectively), but only multiplicity remained significant after multiple-comparison correction. Neonatal seizures likely attributable to a symptomatic DVA were detected in 2/15 patients with c-DVAs. One additional patient with a c-DVA developed probable DVA-related seizures at 11 months. A direct causal relationship
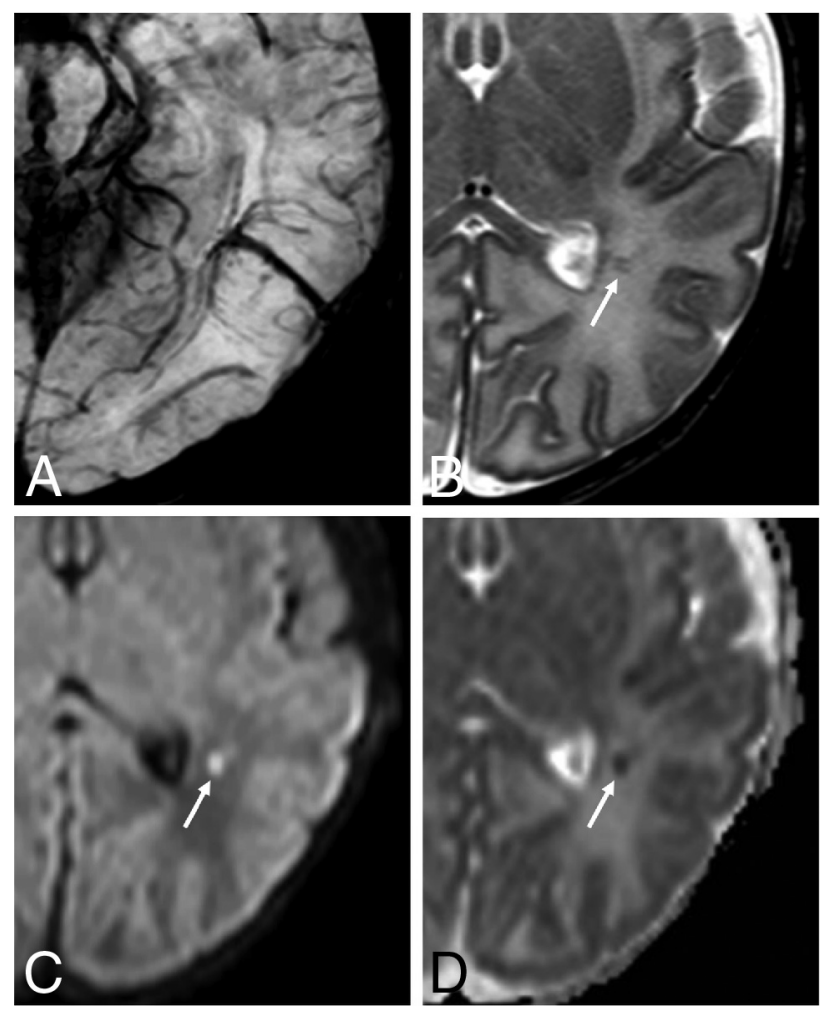

FIG 1. Neonatal developmental venous anomaly complicated by focal areas of venous ischemia. A, Axial SWI shows a left parietal developmental venous anomaly with superficial drainage. $B$, Axial T2WI reveals small linear hypointense lesions in the surrounding WM (arrow), with corresponding hyperintensity on $b=1000$ image (C, arrow) and low ADC values on the ADC map ( $D$, arrow).

Fifteen patients (36.6\%) had at least 1 DVA (range, 1-6) associated with parenchymal abnormalities and/or CSF obstruction (c-DVA group) (Table 1 and Figs 1-3). Two of these neonates between the DVA and neonatal seizures was not identified in 2 patients with u-DVA presenting with this symptom.

Overall, 58 DVAs were identified, comprising multiple DVAs in 9 cases. DVA location and angioarchitecture features are presented in Table 1. Multiple collectors and larger collector calibers were significantly more frequent in complicated DVAs $(P=.008$ and $P<.001$, respectively), even after adjusting for multiple comparisons.

DSA was performed in 4 patients with c-DVAs. No signs of arteriovenous shunting through the DVA with or without an associated classic nidus were identified, while a subject with CVMS had an intraorbital AVF.

\section{Fetal MR Imaging and Postnatal cUS}

Fetal MR imaging was performed in 11 patients $(26.8 \%)$, of whom 6 belonged to the c-DVA group (21 examinations in total, 1-4 studies per patient, acquired between 20 and 38 gestational weeks). Retrospective analysis of single-shot FSE, $b=0$, and/or T2*WI identified a DVA and/or an abnormally enlarged draining pathway in 3 fetuses $(27.3 \%)$. In another case, a DVAassociated cerebellar hemorrhage was detected but precluded the identification of the subjacent DVA. Of the remaining 7 fetuses in whom the DVA was not visible, 3 presented with craniofacial vascular lesions.

Postnatal cUS was available in 36 neonates: In 3 cases, the DVA was suspected before the MR imaging examination due to the presence of a parenchymal linear hyperechogenic focus.

\section{Management and Clinicoradiologic Outcome of Neonates with c-DVAs}

Of 15 neonates with c-DVAs, 13 were conservatively managed, with a wait-and-see approach in 10 cases, anticoagulation treatment in 2, and antiepileptic drugs in 1. Endoscopic third ventriculostomy was performed in 1 neonate with DVA-related obstructive 

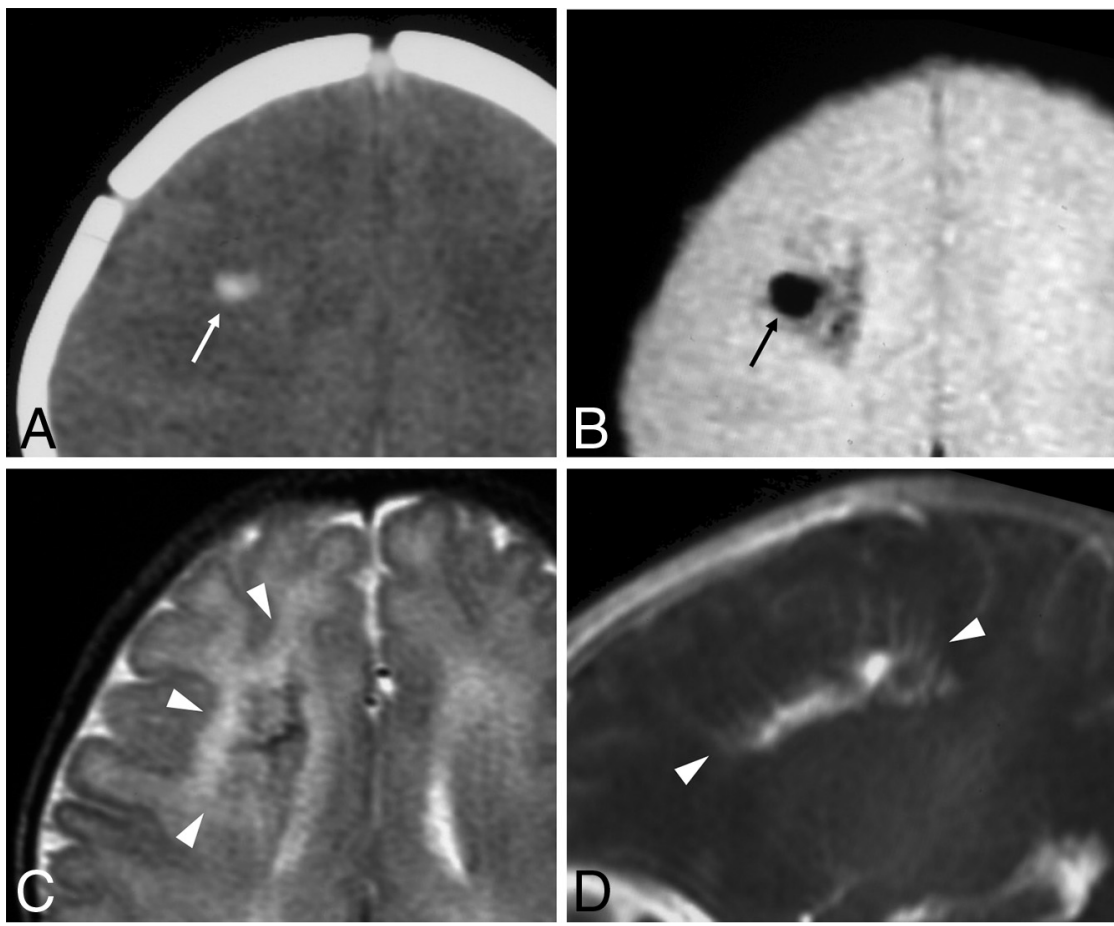

FIG 2. Neonatal developmental venous anomaly complicated by focal hemorrhage and diffuse WM signal abnormalities likely related to venous congestion. $A$, Unenhanced head CT scan demonstrates a focal area of spontaneous hyperdensity (white arrow) in the right frontal region, suggestive of recent hemorrhage. Corresponding axial gradient-echo $\mathrm{T}^{*}$-weighted image $(B)$ and T2WI (C) show a blooming artifact (black arrow) in the region corresponding to the hemorrhage, which subsequently regressed (not shown), and diffuse hyperintensity of the surrounding WM (arrowheads), in keeping with venous congestion. D, Sagittal contrast-enhanced TIWI reveals a large developmental venous anomaly characterized by several radially-oriented dilated veins with a caput medusae morphology and deep venous drainage (arrowheads).

hydrocephalus. Multiple interventional procedures were performed in the child with CVMS and an AVF.

Table 2 reports the clinicoradiologic outcome of subjects with c-DVAs. Longitudinal MR imaging was available in 10/15 patients (median follow-up, 39.1 months; range, 2-97 months; age at last follow-up, 2.5 months-8.2 years). Eight patients (80\%) showed imaging signs of improvement, while stability $(n=1)$ or mixed evolution $(n=1)$ was detected in the remaining cases.

Follow-up neurologic evaluation was available in 14 neonates with c-DVAs (median follow-up, 27.5 months; range, 11-97 months), and findings were judged normal in 9 cases (64.2\%), while minor or moderate psychomotor impairment was detected in $4(28.5 \%)$ and $1(7.1 \%)$ patient, respectively.

A brief description of a few illustrative cases of neonatal cDVA is presented in the On-line Appendix.

\section{DISCUSSION}

In this study, we identified 41 neonates with DVAs, for a total of 58 DVAs, from a population of 2135 neonates undergoing brain MR imaging for diverse clinical reasons and with different imaging techniques, corresponding to a real-world detection in a tertiary pediatric center of $1.9 \%$. These findings are similar to a recent retrospective study by Brinjikji et al, ${ }^{25}$ describing a prevalence of
$1.5 \%$ in the 0 - to 12 -month age group. Most interesting, both percentages are lower than those reported in studies including older children, adults, or mixed populations $(5 \%-10 \%) .{ }^{4,23,25}$ Because the pathogenesis of DVAs remains controversial, including their cause and timing of development, some authors have attributed these age-related prevalence differences to a postnatal origin. ${ }^{25}$ However, caution is advised due to methodologic discrepancies among studies in terms of selection criteria and imaging protocols. In addition, DVAs may potentially be more difficult to detect in neonates due to small head size, incomplete myelination, short imaging protocols, and motion artifacts. On the other hand, statistically significant associations between DVAs and both primary brain tumors and multiple sclerosis have been previously described. ${ }^{18,20}$ Because these disorders are frequent MR imaging indications in adults but very uncommon in the neonatal setting and infancy, the clinical indication itself may act as a confounder in the relationship between age and DVAs. Prospective neuroimaging studies in the healthy population at different ages using standardized imaging protocols are needed to better understand the relationship between age and DVAs. Of note, we retrospectively identified DVAs and/or related enlarged drainage pathways in $27.2 \%$ of cases with available fetal MR imaging, confirming a congenital origin of these vascular abnormalities in those patients. ${ }^{6,27}$ Conversely, we did not identify new DVAs in follow-up studies, but we considered this a limited population; thus, we cannot exclude some DVAs actually developing de novo postnatally.

In our cohort, greater than one-third of neonates presented with at least 1 type of vascular complication directly linked to DVAs. Similarly, Horsch et $\mathrm{al}^{22}$ found a high percentage (42.9\%) of abnormalities surrounding neonatal DVAs, while variable frequencies have been described in studies including adults and/or older children. ${ }^{10-13,15}$ Of note, initial differences regarding the corrected age at first MR imaging, prematurity, and imaging indications between neonates with cDVAs and u-DVAs likely represent a detection bias related to the neuroimaging screening program of preterm neonates with birth weights of $<1500$ g performed in our institution or even by chance, because these values did not reach statistical significance after multiple-comparison correction.

In detail, associated WM signal abnormalities were present in $17.1 \%$ of our neonates and were even more frequent in the series published by Horsch et $\mathrm{al}^{22}$ (21.4\%). Previous studies have suggested that DVA-related WM changes present a bimodal distribution, peaking in younger children and older adults. ${ }^{11,12}$ However, 
the underlying mechanisms of these signal changes remain poorly understood. In younger children, delayed myelination in the draining territory of the DVA has been proposed as a potential explanation. ${ }^{12}$ Alternatively, these signal alterations may represent venous congestion edema in the DVA territory due to an imbalance of the
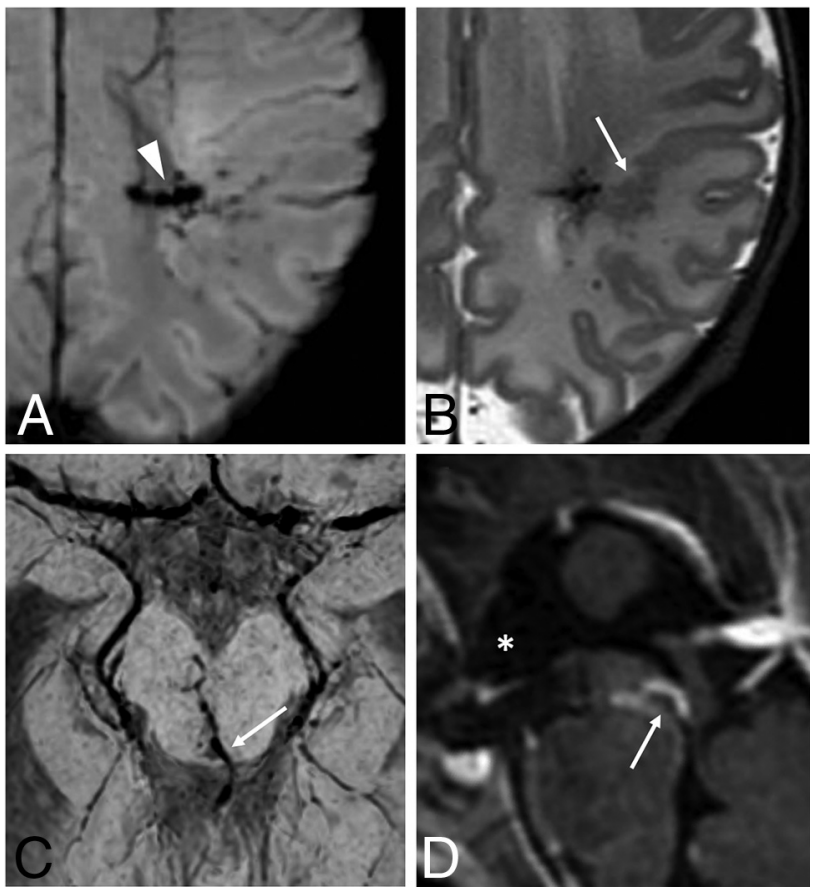

FIG 3. Neonatal developmental venous anomalies associated with focal polymicrogyria $(A$ and $B$ ) and supratentorial hydrocephalus ( $C$ and $D)$ in 2 different patients. Axial SWI $(A)$ and T2WI $(B)$ depict a developmental venous anomaly with deep venous drainage (arrowhead) and an adjacent area of cortical abnormality consistent with focal polymicrogyria (arrow). Axial SWI (C) and sagittal postgadolinium TWWI (D) demonstrate a mesencephalic developmental venous anomaly with the venous collector (arrows) causing focal compression of the inferior third of the cerebral aqueduct and consequent dilation of the anterior recesses of the third ventricle (asterisk), in keeping with supratentorial obstructive hydrocephalus (see also On-line Fig 8).

Table 2: Neuroimaging abnormalities associated with developmental venous anomalies

\begin{tabular}{lcl}
\hline \multicolumn{1}{c}{ MRI Abnormalities } & $\begin{array}{c}\text { Neonatal Period } \\
(\boldsymbol{n}=\mathbf{1 5})\end{array}$ & \multicolumn{1}{c}{ Last Follow-Up ${ }^{\mathrm{a}}(\boldsymbol{n}=10)$} \\
\hline $\begin{array}{l}\text { WM T2 signal } \\
\text { abnormalities }\end{array}$ & 7 & $\begin{array}{l}\text { Reduced } 2 / 5 \\
\text { Stable } 1 / 5 \\
\text { Complete regression, } 2 / 5\end{array}$ \\
$\begin{array}{l}\text { Restricted diffusion foci } \\
\text { Hemorrhagic foci }\end{array}$ & 4 & $\begin{array}{l}\text { Total regression, } 2 / 2 \\
\text { Gliosis with or without hemosiderin } \\
\text { deposits, } 3 / 3\end{array}$ \\
Multiple CCM & 8 & $\begin{array}{l}\text { Stable, } 1 / 2 \\
\text { Growth, } 1 / 2\end{array}$ \\
$\begin{array}{l}\text { PMG } \\
\text { Calcifications }\end{array}$ & $2^{\text {b }}$ & $\begin{array}{l}\text { Stable, } 2 / 2 \\
\text { Triventricular } \\
\text { hydrocephalus }\end{array}$ \\
Draining venous varix \\
thrombosis
\end{tabular}

Note:-PMG indicates polymicrogyria.

a Some patients presented with $\geq 1$ DVA-related complication.

${ }^{b}$ Includes 1 neonate with cerebrofacial venous metameric syndrome.

${ }^{\text {c }}$ Postendoscopic third ventriculostomy. in- and outflow of blood in the DVA system, raising the pressure in the DVA. ${ }^{8}$ Of note, the latter mechanisms can also explain the relatively high frequency of associated hemorrhages and/or ischemic changes identified in our sample $(19.5 \%$ and $9.8 \%$ of cases, respectively). In the general population, the risk of DVA-related hemorrhage is considered to be low ( $<1 \%$ /year) and is usually attributed to adjacent CCM bleeding. ${ }^{2}$ However, we detected CCMs in only a small percentage of cases (4.9\%), in keeping with the theory that nonfamilial CCMs are acquired lesions related to DVAs through the process of hemorrhagic angiogenic proliferation. ${ }^{28,29}$

Taken together, our findings suggest that in the neonatal period, there is a higher risk of flow-related complications in DVAs, potentially leading to venous hypertension and associated venous congestion, hemorrhage, and/or infarction. Putative neonatal risk factors of hemodynamic decompensation include mechanical distortion during vaginal birth and immaturity of the venous, immune, and hemostatic systems as well as hypercoagulability, which may be potentiated by maternal factors or inflammation. ${ }^{30-32}$ Finally, angioarchitectural factors yet unexplored in the neonatal setting, including angulation and stenosis of draining veins or tortuosity of medullary veins, could contribute to the development of ischemic or hemorrhagic complications. ${ }^{10,33}$

Most interesting, the presence of multiple DVAs (ie, multiplicity) was significantly more common in neonates with c-DVAs, even after multiple-comparisons correction, suggesting that more severe and widespread venous pathology may correspond to a more fragile venous outflow system and/or a higher propensity for thrombotic DVA events. Of note, 2 of these neonates presented with clinical-neuroradiologic features consistent with CVMS, a rare craniofacial vascular malformation disorder characterized by a wide spectrum of slow-flow vascular lesions distributed along $\geq 1$ of the 3 craniofacial metameres, further supporting this theory. ${ }^{19}$ Remarkably, 1 neonate also presented with a superior orbital fissure AVF, suggesting that this complex disorder may actually be a continuum potentially affecting $>1$ vessel type.

Our study also revealed focal polymicrogyria in the draining region of a DVA in 2 neonates (4.9\%). DVAs or other venous drainage abnormalities or both have already been described adjacent to dysplastic cortical areas using conventional and ultra-high-field MR imaging. ${ }^{34-37}$ Because polymicrogyria is frequently associated with in utero disruptive events, coexistence of these 2 lesions suggests a causative effect of the DVA in the formation of this cortical malformation or, more probably, a shared pathomechanism related to early failure, abnormal development, or intrauterine occlusion of normal cerebral vessels. $^{34,35,38}$ Finally, in 1 neonate, we observed obstructive hydrocephalus related to another type of DVA complication, ie, mechanical compression of the cerebral aqueduct. ${ }^{8}$ As in our patient, CSF diversion techniques usually lead to a good outcome in these rare cases. ${ }^{39}$ 
As previously described, in our neonatal cohort, DVAs were more commonly located supratentorially and in the frontal lobe (41.4\%). ${ }^{10,32}$ Other common locations included the parieto-occipital (27.7\%) and temporal (13.8\%) lobes, while the basal ganglia and thalamus were involved in only $8.6 \%$ of cases. Of note, different from a previous neonatal case series, in our series, we identified infratentorial DVAs in $8.4 \%$ of cases, thus confirming a potential selection bias related to the use of cUS to depict posterior fossa DVAs. ${ }^{22}$

Regarding angioarchitecture features, we noticed a higher prevalence of multiple DVA collectors, which, together with larger caliber collectors, were significantly associated with DVArelated parenchymal abnormalities. These features may be related to the DVA size and, ultimately, to the volume of parenchyma under hemodynamic stress, ie, with reduced venous drainage capacity. Larger collectors may also be theoretically more prone to abnormal venous flow, with increased stasis and thrombosis. However, other studies performed in adults did not show statistically significant differences between parenchymal abnormalities and collecting vein diameters; ${ }^{11}$ therefore, the relationship between these neuroimaging features requires more detailed study. Similarly, in the present study, posterior fossa location was not a risk factor for complicated DVAs. Methodologic issues in terms of populations of interest and types of complication may justify this variability, ${ }^{16,23,32}$ and further studies are needed to also address this topic.

Serial imaging of a subgroup of neonates with c-DVAs revealed that DVAs and adjacent MR imaging abnormalities frequently present a dynamic evolution during the early years of life. These findings are in line with previous studies and probably reflect progressive brain and vascular maturation during early infancy. ${ }^{22,40}$ Indeed, neuroimaging follow-up demonstrated overall improvement in most cases of c-DVAs. More specifically, WM abnormalities were reduced in size or even completely resolved. Furthermore, ischemic and hemorrhagic foci also tended to subside without signs of intracranial re-hemorrhage. Of note, clinical outcomes of patients with c-DVAs was concordant with their favorable MR imaging evolution, with normal neurologic examination findings in most cases. Good clinical and neurologic outcomes were also reported by Horsch et $\mathrm{al}^{22}$ and are probably related to intrinsic brain plasticity as well as normalization of potential risk factors present in the neonatal phase.

\section{Limitations}

This study has some limitations. First, case selection was based on a retrospective single-center search of radiology reports. Therefore, although DVAs are routinely described in our institution by all staff members, the true DVA prevalence might be underestimated. Similarly, a relevant number of neonates was scanned on a $1.5 \mathrm{~T}$ system, and gadolinium-based contrast media were only occasionally used, potentially leading to lower DVA detection. ${ }^{34}$ However, SWI was performed in almost all neonates (92.7\%) and has a high diagnostic sensitivity for DVAs in children, especially when sedation is achieved without propofol and sevoflurane. ${ }^{4}$ Second, this study was performed in a tertiary pediatric institution, leading to potential selection bias toward inclusion of more severe DVA cases and limiting generalizability toward a different setting. Moreover, DVA collectors were measured on axial $\mathrm{T} 2^{\star} \mathrm{WI}$, and this sequence can be influenced by the level of blood oxygenation and the magnetic field strength. However, none of the neonates were examined under general anesthesia, and complicated DVAs were actually less frequent in the group of subjects scanned using a 3T magnet. Therefore, if there were any bias related to the examination technique in terms of DVA collector size and MR imaging complications, it would actually exert its influence toward the null hypothesis. Finally, longitudinal data were missing in some patients, and clinical evaluation at follow-up was obtained from clinical records, though formal neurologic evaluation was performed in all assessed cases.

\section{CONCLUSIONS}

Real-world DVA detection in this population of neonates with clinically-indicated brain MR imaging reached $1.9 \%$, which is lower than percentages of studies including older children and adults and might be an underestimation of the true prevalence. Of all neonates with detected DVAs, around one-third presented with DVA-related complications. The latter group had a significant tendency toward multiplicity and additional vascular malformations but usually had favorable neuroimaging findings and neurologic evolution at follow-up. DVAs could be retrospectively diagnosed in utero in one-quarter of neonates with fetal MR imaging, confirming, at least in these cases, a congenital origin.

Disclosures: Ana F. Geraldo-UNRELATED: Grants/Grants Pending: European Society of Neuroradiology, Comments: annual research grant. Alessandro Parodi-UNRELATED: Consultancy: Shire Human Genetic Therapies, Comments: 2018-2019 collaboration in the ROPP-2008-01 clinical trial (assessment of cranial ultrasound images of enrolled subjects). Paul Govaert-UNRELATED: Payment for Development of Educational Presentations: book, Mac Keith Press London. Mariasavina Severino-RELATED: Grant: "Ricerca Corrente Disordini Neurologici e Muscolari (Linea 5)" of Italian Ministry of Health, Compagnia di San Paolo (ROL 20573)*; UNRELATED: Employment: neuroradiology consultant, Scientific Institute for Research, Hospitalization and Healthcare Istituto Giannina Gaslini. *Money paid to the institution.

\section{REFERENCES}

1. Malova M, Rossi A, Severino M, et al. Incidental findings on routine brain MRI scans in preterm infants. Arch Dis Child Fetal Neonatal Ed 2017;102:F73-78 CrossRef Medline

2. Hon JM, Bhattacharya JJ, Counsell CE, et al; SIVMS Collaborators. The presentation and clinical course of intracranial developmental venous anomalies in adults: a systematic review and prospective, population-based study. Stroke 2009;40:1980-85 CrossRef Medline

3. Lee C, Pennington MA, Kenney CM, et al. MR evaluation of developmental venous anomalies: medullary venous anatomy of venous angiomas. AJNR Am J Neuroradiol 1996;17:61-70 Medline

4. Gökçe E, Acu B, Beyhan M, et al. Magnetic resonance imaging findings of developmental venous anomalies. Clin Neuroradiol 2014;24:135-43 CrossRef Medline

5. Young A, Poretti A, Bosemani T, et al. Sensitivity of susceptibilityweighted imaging in detecting developmental venous anomalies and associated cavernomas and microhemorrhages in children. Neuroradiology 2017;59:797-802 CrossRef Medline

6. Geraldo AF, Melo M, Monteiro D, et al. Developmental venous anomaly depicted incidentally in fetal MRI and confirmed in postnatal MRI. Neuroradiology 2018;60:993-94 CrossRef Medline

7. Lasjaunias $P$, Burrows $P$, Planet C. Developmental venous anomalies (DVA): the so-called venous angioma. Neurosurg Rev 1986;9:233-42 CrossRef Medline 
8. Pereira VM, Geibprasert S, Krings T, et al. Pathomechanisms of symptomatic developmental venous anomalies. Stroke 2008;39:320115 CrossRef Medline

9. Rinaldo L, Lanzino G, Flemming KD, et al. Symptomatic developmental venous anomalies. Acta Neurochir (Wien) 2020;162:1115-25 CrossRef Medline

10. San Millán Ruíz D, Delavelle J, Yilmaz H, et al. Parenchymal abnormalities associated with developmental venous anomalies. Neuroradiology 2007;49:987-95 CrossRef Medline

11. Santucci GM, Leach JL, Ying J, et al. Brain parenchymal signal abnormalities associated with developmental venous anomalies: detailed MR imaging assessment. AJNR Am J Neuroradiol 2008;29:1317-23 CrossRef Medline

12. Linscott LL, Leach JL, Zhang B, et al. Brain parenchymal signal abnormalities associated with developmental venous anomalies in children and young adults. AJNR Am J Neuroradiol 2014;35:160007 CrossRef Medline

13. Takasugi M, Fujii S, Shinohara Y, et al. Parenchymal hypointense foci associated with developmental venous anomalies: evaluation by phase-sensitive MR imaging at 3T. AJNR Am J Neuroradiol 2013;34:1940-44 CrossRef Medline

14. Sharma A, Zipfel GJ, Hildebolt C, et al. Hemodynamic effects of developmental venous anomalies with and without cavernous malformations. AJNR Am J Neuroradiol 2013;34:1746-51 CrossRef Medline

15. Umino $M$, Maeda $M$, Matsushima $N$, et al. High-signal-intensity abnormalities evaluated by $3 \mathrm{D}$ fluid-attenuated inversion recovery imaging within the drainage territory of developmental venous anomalies identified by susceptibility-weighted imaging at $3 \mathrm{~T}$. Jpn J Radiol 2014;32:397-404 CrossRef Medline

16. Zhang S, Ma L, Wu C, et al. A rupture risk analysis of cerebral cavernous malformation associated with developmental venous anomaly using susceptibility-weighted imaging. Neuroradiology 2020;62:39-47 CrossRef Medline

17. Jones BV, Linscott L, Koberlein G, et al. Increased prevalence of developmental venous anomalies in children with intracranial neoplasms. AJNR Am J Neuroradiol 2015;36:1782-85 CrossRef Medline

18. Roux A, Edjlali M, Porelli S, et al. Developmental venous anomaly in adult patients with diffuse glioma: a clinically relevant coexistence? Neurology 2019;92:e55-62 CrossRef Medline

19. Brinjikji W, Nicholson $\mathrm{P}$, Hilditch $\mathrm{CA}$, et al. Cerebrofacial venous metameric syndrome: spectrum of imaging findings. Neuroradiology 2020;62:417-25 CrossRef Medline

20. Halicioglu S, Turkoglu SA. Role of developmental venous anomalies in etiopathogenesis of demyelinating diseases. Int $J$ Neurosci 2019;129:245-51 CrossRef Medline

21. Shiran SI, Ben-Sira L, Elhasid R, et al. Multiple brain developmental venous anomalies as a marker for constitutional mismatch repair deficiency syndrome. AJNR Am J Neuroradiol 2018;39:1943-46 CrossRef Medline

22. Horsch S, Govaert P, Cowan FM, et al. Developmental venous anomaly in the newborn brain. Neuroradiology 2014;56:579-88 CrossRef Medline

23. Silva $\mathrm{AH}$, Wijesinghe $\mathrm{H}$, Lo WB, et al. Paediatric developmental venous anomalies (DVAs): how often do they bleed and where? Childs Nerv Syst 2020;36:1435-43 CrossRef Medline
24. Linscott LL, Leach JL, Jones BV, et al. Developmental venous anomalies of the brain in children: imaging spectrum and update. Pediatr Radiol 2016;46:394-406 CrossRef Medline

25. Brinjikji W, El-Masri AE, Wald JT, et al. Prevalence of developmental venous anomalies increases with age. Stroke 2017;48:1997-99 CrossRef Medline

26. Zabramski JM, Wascher TM, Spetzler RF, et al. The natural history of familial cavernous malformations: results of an ongoing study. $J$ Neurosurg 1994;80:422-32 CrossRef Medline

27. Okudera T, Huang YP, Fukusumi A, et al. Micro-angiographical studies of the medullary venous system of the cerebral hemisphere. Neuropathology 1999;19:93-118 CrossRef Medline

28. Dammann $\mathrm{P}$, Wrede $\mathrm{K}$, Zhu $\mathrm{Y}$, et al. Correlation of the venous angioarchitecture of multiple cerebral cavernous malformations with familial or sporadic disease: a susceptibility-weighted imaging study with 7-Tesla MRI. J Neurosurg 2017;126:570-77 CrossRef Medline

29. Brinjikji W, El-Masri AE, Wald JT, et al. Prevalence of cerebral cavernous malformations associated with developmental venous anomalies increases with age. Childs Nerv Syst 2017;33:1539-43 CrossRef Medline

30. Yang JY, Chan AK, Callen DJ, et al. Neonatal cerebral sinovenous thrombosis: shifting the evidence for a diagnostic plan and treatment strategy. Pediatrics 2010;126:e693-e700 CrossRef Medline

31. Ami O, Maran JC, Gabor P, et al. Three-dimensional magnetic resonance imaging of fetal head molding and brain shape changes during the second stage of labor. PLoS One 2019;14:e0215721 CrossRef Medline

32. Kumar S, Lanzino G, Brinjikji W, et al. Infratentorial developmental venous abnormalities and inflammation increase odds of sporadic cavernous malformation. J Stroke Cerebrovasc Dis 2019;28:1662-67 CrossRef Medline

33. Hong YJ, Chung TS, Suh SH, et al. The angioarchitectural factors of the cerebral developmental venous anomaly: can they be the causes of concurrent sporadic cavernous malformation? Neuroradiology 2010;52:883-91 CrossRef Medline

34. Thompson JE, Castillo M, Thomas D, et al. Radiologic-pathologic correlation polymicrogyria. AJNR Am J Neuroradiol 1997;18:307-12 Medline

35. Cagneaux M, Paoli V, Blanchard G, et al. Pre- and postnatal imaging of early cerebral damage in Sturge-Weber syndrome. Pediatr Radio 2013;43:1536-39 CrossRef Medline

36. De Ciantis A, Barkovich AJ, Cosottini M, et al. Ultra-high-field MR imaging in polymicrogyria and epilepsy. AJNR Am J Neuroradiol 2015;36:309-16 CrossRef Medline

37. Mankad K, Biswas A, Espagnet MCR, et al. Venous pathologies in paediatric neuroradiology: from foetal to adolescent life. Neuroradiology 2020;62:15-37 CrossRef Medline

38. Stutterd CA, Leventer RJ. Polymicrogyria: a common and heterogeneous malformation of cortical development. Am J Med Genet $C$ Semin Med Genet 2014;166C:227-39 CrossRef Medline

39. Higa N, Dwiutomo R, Oyoshi T, et al. A case of developing obstructive hydrocephalus following aqueductal stenosis caused by developmental venous anomalies. Childs Nerv Syst 2020;36:1549-55 CrossRef Medline

40. Howard T, Abruzzo T, Jones B, et al. Postnatal evolution of a developmental venous anomaly. J Pediatr Neuroradiol 2015;01:305-11 CrossRef 